\title{
Experimental Model of Non-Controlled Hemorrhagic Shock in Pigs
}

\author{
Fernanda Paula Cavalcante ${ }^{1}$, Ricardo Souza Nani, TSA ${ }^{2}$, Joel Avancini Rocha Filho ${ }^{3}$, \\ José Otávio Costa Auler Junior ${ }^{4}$, Maria José Carvalho Carmona ${ }^{5}$, Marcel Cerqueira Cesar Machado ${ }^{6}$
}

Summary: Cavalcante FP, Nani RS, Rocha Filho JA, Auler Junior JOC, Carmona MJC, MachadoMCC - Experimental Model of Non-Controlled Hemorrhagic Shock in Pigs.

Background and objectives: A better understanding of pathophysiologic changes associated to trauma and hemorrhagic shock can help the development of therapies capable of reducing trauma-related mortality. The objective of this study was to describe a model of non-controlled hemorrhagic shock in pigs.

Methods: Animals received ketamine and midazolam as pre-anesthetic medications. Anesthesia was induced with propofol, and tracheal intubation was performed with the animals on spontaneous ventilation. After intubation neuromuscular blockade was performed. Animals were maintained in controlled mechanical ventilation and normocapnia. Anesthesia was maintained with propofol and fentanyl as needed. Saline was infused during the entire preparation period.

Monitoring: Cardioscope, pulse oximeter, invasive blood pressure, volumetric catheter in the pulmonary artery, and urine output by cystostomy were used. Experimental model: after the initial recording of hemodynamic, metabolic, and coagulation variables, right subcostal incision and left lobe liver biopsy were performed. Anesthetic infusion was reduced while the infusion of saline was interrupted. An incision $12 \mathrm{~cm}$ long $2 \mathrm{~cm}$ deep was performed in the right liver lobe followed by digital divulsion of the wound. During the hemorrhagic phase, an aspiration probe was placed close to the wound and the volume of aspirated blood was recorded. When mean arterial pressure reached $40 \mathrm{mmHg}$ and bleeding was above $700 \mathrm{~mL}$ the intervention phase was initiated according to the type of study.

Conclusion: The development of experimental models to reduce high mortality and costs related to trauma is important.

Keywords: Abdomen; Wounds and Injuries; Shock, Hemorrhagic; Models, Animal.

๑2011 Elsevier Editora Ltda. All rights reserved.

\section{INTRODUCTION}

According to IBGE data, external mortality-related causes are the third cause of general mortality in Brazil behind only of deaths from cardiovascular causes and malignant neoplasia. It is the main cause of death of individuals of both genders with ages from 1 to 44 years. In the United States, traumarelated costs are comparable to the costs associated with malignant neoplasia, reaching US $\$ 44$ billion in $1997^{1}$.

The association of trauma, hemorrhage, hemorrhagic shock, organ ischemia, volume resuscitation, and reperfusion lesions causes local and systemic alterations. Even those

Received from Faculdade de Medicina da Universidade de São Paulo (USP), Brazil.

1. R3 CET/SBA, Faculdade de Medicina da Universidade de São Paulo (USP)

2. Assistant Physician of Anesthesiology Division of Hospital das Clínicas da Faculdade de Medicina da USP;

3. PhD; Assistant Physician of Anesthesiology Division of Hospital das Clínicas da Faculdade de Medicina da USP

4. PhD;Full Professor of Anesthesiology of Faculdade de Medicina da USP

5. PhD; Associate Professor of Anesthesiology of Faculdade de Medicina da USP

6. PhD; Professor Emeritus of Faculdade de Medicina da USP

Submitted on January 4, 2011

Approved on February 21, 2011

Correspondence to:

Dra. Fernanda Paula Cavalcante

Av. Sen. Virgilio Távora, 77 Apt 803

60170250 - Fortaleza, CE, Brazil

E-mail: cavalcantefp@yahoo.com.br who survive the initial phase of hemorrhagic shock still have a risk of death from sepsis and/or multiple organ dysfunction ${ }^{1,2}$. Better understanding of trauma and hemorrhagic shock-related pathophysiology and immunologic changes can help the development of new therapies capable of reducing traumarelated mortality and costs 1,2 .

Several experimental models have been developed in the last few decades in an attempt to investigate the pathophysiology of hemorrhagic shock and to validate new therapies ${ }^{1-3}$. Even consisting in its majority in models that are difficult to reproduce in laboratory that also do not reproduce real clinical scenery, experimental models have the advantage of producing information about well-defined situations that can be useful in the development of new therapies to reduce traumarelated mortality ${ }^{1-3}$.

The objective of the present study was to describe a noncontrolled hemorrhagic shock model in pigs, developed to investigate strategies of volume replacement in pre-hospital environment.

\section{METHODS}

After approval by the Ethics Commission for Analysis of Research Studies (CAPPesq HCFMUSP), the experimental 
mode was developed with 32 healthy adult swine of the Penarlan race weighing 25 to $35 \mathrm{~kg}$ at the Laboratório de Investigação Médica of the Disciplina de Cirurgia e Transplante de Fígado and at the Laboratório of the Disciplina de Anestesiologia of Faculdade de Medicina of Universidade de São Paulo. Qualified personnel supervised by veterinarians were responsible for the caring of animals according to current recommendations. At the end of the study, the animals were discarded according to the rules of the sanitary vigilance. On the night before the experiment, animals did not have access to solid foods, but access to water was maintained.

\section{Experimental protocol}

\section{Anesthesia}

Animals received ketamine $\left(0.5 \mathrm{mg} \cdot \mathrm{kg}^{-1} \mathrm{IM}\right)$ and midazolam (0.1 mg. kg-1 IM) 30 minutes before the surgery as pre-anesthetic medication. Body temperature was maintained above $38 \mathrm{C}$ with a thermal blanket throughout the procedure. After catheterizing the left auricular vein with a $20 \mathrm{G}$ Teflon ${ }^{\circledR}$ catheter, anesthesia was induced with propofol (1-2 mg.kg-1 EV), and tracheal intubation was performed while animals were breathing spontaneously. After tracheal intubation, neuromuscular blockade was performed with $8 \mathrm{mg}$ of pancuronium. Animals were maintained on controlled mechanical respiration (Servo Siemens $900 \mathrm{C}$ ) with $\mathrm{FiO}_{2}$ of $35 \%$, respiratory rate 20 breaths per minute, and tidal volume adjusted to maintain normocapnia. Anesthesia was maintained with propofol (100$\left.140 \mu \mathrm{g} \cdot \mathrm{kg}^{-1} \cdot \mathrm{min}^{-1}\right)$ and fentanyl $\left(5 \mu \mathrm{g} \cdot \mathrm{kg}^{-1}\right)$ as needed. Saline was infused IV $\left(6 \mathrm{~mL} . \mathrm{kg}^{-1} \cdot \mathrm{h}^{-1}\right)$ throughout the preparation period.

\section{Monitoring}

Cardioscope on three derivations, though analyzed on D2, and pulse oximetry were used in non-invasive monitoring during the procedure.

Invasive monitoring was accomplished with a volumetric pulmonary catheter installed by right cervical vein dissection for continuous monitoring of pulmonary artery oxygen saturation. A 5F arterial catheter installed by arterial dissection of the left inguinal artery was used for continuous measurement of invasive blood pressure. A 14G venous catheter for volume replacement, and a \#12 vesical catheter, by cystostomy, to measure urine output were also placed.

\section{Experimental model}

This is a non-controlled hemorrhagic shock model.

After initial recording of hemodynamic, metabolic, and coagulation variables, a right subcostal abdominal incision and a left lobe hepatic biopsy were performed.
Propofol infusion was reduced to $33 \mu \mathrm{g} \cdot \mathrm{kg}^{-1} \cdot \mathrm{min}^{-1}$, while isotonic saline infusion was interrupted to minimize the anesthetic and volume replacement effects.

An incision $12 \mathrm{~cm}$ long and $2 \mathrm{~cm}$ deep was performed in the right liver lobe, followed by digital divulsion of the wound. In the hemorrhagic phase an aspiration tube was placed close to the hepatic incision and the volume of blood aspirated was recorded.

When mean arterial pressure reached $40 \mathrm{mmHg}$ and/or bleeding was higher than $700 \mathrm{~mL}$, it was possible to initiate the proposed intervention phase according to the objective of the study.

\section{Variables analyzed}

Through this experimental model it was possible to analyze hemodynamic variables such as pressures, indexes, and vascular resistances. Oxygenation and metabolism data, such as arterial and venous blood gases, lactate, and glucose levels, as well as coagulation data, such as d-dimer, prothrombin time, thrombin time, partial thromboplastin time, thrombomodulin, C protein, fibrinogen, platelet count, and thromboelastometry, were analyzed. Urine output and bleeding volume were quantified.

\section{CONCLUSIONS}

Due to the great variety of available experimental models, the difficulty to reproduce the models in laboratories, and the inevitable questioning on its clinical applicability, investigators should carefully chose the best model possible according to their objective. An experimental model for trauma and hemorrhagic shock capable of answering all questions on the subject is not available ${ }^{3}$. Producing an ideal model capable of simulating real clinical situations and to elucidate therapeutic strategies applicable to daily medical practice is difficult. Each investigator should use and perfect the model that shows more adaptation to his objective ${ }^{2}$. When choosing the experimental model the definition of the adequate animal, among other things, is important. In general, rats are better for biochemical studies, while others are better adapted to studies on hemodynamic changes ${ }^{2}$. Variations in the response to trauma and the therapy among the different species should be taken into consideration when extrapolating the results to clinical practice.

Even with the difficulty of transposing experimental results to clinical practice, it is important to continue the development of experimental studies on hemorrhagic shock, since the conclusions of these studies are relevant and certainly can improve clinical pre-hospital and hospital performance to reduce the high mortality rates and costs associated to trauma. 